\title{
Plastic versus glass capillaries for rapid-cycle PCR
}

\author{
Oluwole Elenitoba-Johnson ${ }^{1}$, Derek David ${ }^{2}$, Niel Crews ${ }^{1}$, and \\ Carl T. Wittwer ${ }^{1}$ \\ 1 University of Utah, Salt Lake City and 'Idaho Technology, Salt Lake City, Utah, USA
}

BioTechniques 44:487-492 (April 2008)

doi $10.2144 / 000112722$

Rapid-cycle PCR uses fast temperature transitions and minimal denaturation and annealing times of " 0 " s to complete 30 cycles in 10 to $30 \mathrm{~min}$. The most popular platform amplifies samples in glass capillaries arranged around a carousel with circulating air for temperature control. Recently, plastic capillary replacements for glass capillaries became available. We compared the performance of plastic and glass capillaries for rapid-cycle PCR. Heat transfer into plastic capillaries was slowed by thicker walls, lower thermal conductivity, and a lower surface area-to-volume ratio than glass capillaries. Whereas the denaturation and annealing target temperatures were reached by samples in glass capillaries, samples in plastic capillaries fell short of these target temperatures by $6^{\circ}-7^{\circ} \mathrm{C}$. Rapid-cycle PCR was performed on two human genomic targets (APOE and ACVRL1) and one plasmid (pBR322) to amplify fragments of 225-300 bp in length with melting temperatures of $90.3^{\circ}-93.1^{\circ} \mathrm{C}$. Real-time amplification data, end-point melting curves, and end-point gel analysis revealed strong, specific amplification of samples in glass and complete amplification failure in plastic. Only the APOE target was successfully amplified by extending the denaturation and annealing times to 5 or 10 s. A 20 s holding period was necessary to reach target temperatures in plastic capillaries.

\section{INTRODUCTION}

Rapid-cycle PCR in 10-30 min with cycle times of 20-60 s was first demonstrated in the early 1990s $(1,2)$. Temperature is controlled with circulating air and samples are contained in glass capillary tubes. Momentary denaturation and annealing times of " 0 " $s$ are entirely adequate for PCR $(3,4)$. Furthermore, short annealing times and faster transitions increase specificity without compromising yield (5).

However, rapid-cycle PCR requires accurate control of the sample temperature, not common in most commercial instruments when faster protocols are attempted. As long as the sample temperature actually reaches the denaturation and annealing targets, there is no need for temperature holds to allow the sample temperature to "catch up." Samples within glass capillaries heated by circulating air achieve accurate temperature control by matching the thermal response of the sample in a capillary to a tubular thermocouple that monitors the temperature.

Recently, plastic capillaries became available for use in real-time instruments that were originally designed for rapid cycling with glass capillaries (6). Because of the different thermal characteristics of such tubes, their effect on thermal cycling and PCR amplification with rapid cycling protocols was investigated.

\section{MATERIALS AND METHODS}

\section{Instrumentation and Temperature Monitoring}

A rapid-cycle, real-time $\mathrm{PCR}$ instrument (LightCycler 1.5, Roche, Indianapolis, IN, USA) was used for all studies. Standard glass capillary tubes (20 $\mu \mathrm{L}$ maximum volume) and original carousel (Roche) were compared with plastic capillaries with their matching carousel (SensiTube system, Osmetech, Rockland, MA, USA). The sample temperature during cycling was monitored by inserting a miniature thermocouple (5TC-TT-J-40-36, Omega Engineering, Stamford, CT, USA) into $10 \mu \mathrm{L}$ of water in plastic or glass capillaries so that the thermocouple tip was at the middle of the fluid column. This thermocouple was used in the original development of rapid-cycle PCR $(1,2)$ and is so small ( $0.08 \mathrm{~mm}$ in diameter) that its effect on thermal response is minimal.
The capillary tubes were closed with Critoseal (Kendall, Mansfield, MA, USA) instead of the plastic caps supplied by the manufacturers, in order to avoid thermocouple damage. Temperature data was acquired with a handheld thermometer (Fluke Model 54, Series П, Fluke, Everett, WA, USA) at a rate of once each second and transferred into Excel (Microsoft, Redmond, WA, USA) with an infrared data receiver (XTNDAccess IrDA PC Adapter, Extended Solutions, Boise, ID, USA). Temperature accuracy of this system was within $\pm 0.2^{\circ} \mathrm{C}$ when measured by ice water and boiling water baths.

\section{Physical Tube Measurements}

To determine tube dimensions, glass capillaries were scored and broken halfway down their length. Because the plastic capillaries were tapered, crosssections near the base and tip were obtained by grinding them down on a polishing wheel (M-Prep 3, Allied High Tech Products, Rancho Dominguez, CA, USA) with 600 -grit paper. A CCD camera (Fire-i 400, Unibrain, San Ramon, CA, USA) was used to image the crosssections, the dimensions determined in pixels (QCapture Pro, QImaging, Surrey, BC, Canada) and converted to $\mathrm{mm}$ by correlation to a digital caliper measurement of the outer diameter.

\section{Rapid-Cycle, Real-time PCR}

Three templates were amplified by rapid-cycle PCR. A 225 bp fragment of ACVRL1 (GenBank NC_000012.10) with $68 \%$ GC content was amplified from human genomic DNA using primers 5'-ATCTAACTGGCAGAGTGGTC-3' and 5'-GGCCACGCTGCTTCTC-3' (7). A 277 bp fragment of $A P O E$ (GenBank NC_000019.8) with 75\% GC content was amplified from human genomic DNA using primers 5'-ACGCGG GCACGGCTGTCCAAGG-3' and 5'-GG CGCTCGCGGATGGCGCTGA-3' (8). A 300 bp fragment of pBR322 (GenBank J01749) with 61\% GC content was amplified from a plasmid of the DNA Toolbox (9) (kindly provided by Lonza, Rockland, ME, USA) using primers 5'-CGCACTTATGACTGTC TTCT- ${ }^{\prime}$ and $5^{\prime}$-CCGGAAGCGAGAA GAATCAT-3'. 
PCR was performed in $10 \mu \mathrm{L}$ volumes within either glass or plastic capillaries. Included in the reactions were $2.5 \mathrm{mM}$ $\mathrm{Mg}^{2+}, 50 \mathrm{mM}$ Tris-HCl (pH 8.3), 500 $\mu \mathrm{g} / \mathrm{mL}$ BSA, $200 \mu \mathrm{M}$ of each deoxynucleotide triphosphate, $0.5 \mu \mathrm{M}$ each of two primers, $0.4 \mathrm{U}$ of KlenTaq1 polymerase (Ab Peptides, St. Louis, MO, USA), $88 \mathrm{ng}$ of TaqStart antibody (ClonTech, Mountain View, CA, USA), $1 \times$ LCGreen Plus (Idaho Technology, Salt Lake City, UT, USA), and template DNA. Template DNA was either $1.5 \times 10^{4}$ copies of human genomic DNA or $10^{5}$ copies of plasmid per reaction. Rapid cycling protocols with programmed temperature transitions of $20^{\circ} \mathrm{C} / \mathrm{s}$ were used as specified in each figure legend.

\section{Product Analysis}

After PCR, melting curves were obtained on a high-resolution melting instrument (HR-1; Idaho Technology) at a rate of $0.3^{\circ} \mathrm{C} / \mathrm{s}$ between $75^{\circ}$ and $95^{\circ} \mathrm{C}$ and a fixed LED power of $21.5 \%$. Because the plastic capillary dimensions are different from those of glass capillaries, the standard HR-1 cylindrical heating ingot was replaced with a custom ingot machined out of aluminum to closely fit the plastic capillaries. After melting, PCR products from both plastic and glass capillaries were analyzed on $1.5 \%$ agarose gels.

\section{RESULTS AND DISCUSSION}

A plastic capillary system, including substitute capillaries and a carousel for real-time PCR, was evaluated for rapidcycle PCR. Table 1 compares the dimensions and thermal properties of glass and plastic capillaries. In contrast to straight glass capillaries, plastic capillaries are tapered to allow injection molding, which also results in greater variation in wall thickness. Wall thickness variation was $33 \%$ near the base and $59 \%$ near the tip of the plastic capillaries, compared with $7 \%$ for glass capillaries. The average dimensions of the plastic capillaries were greater than glass capillaries, including the outer diameter (148\%), inner diameter (143\%), and the wall thickness (165\%), leading to a 23\% decrease in surface area-to-volume ratio. The thermal conductivity of the plastic material used is about 7-fold less than glass. Surface area-to-volume ratio, wall thickness, and thermal conductivity all suggest that plastic capillaries will respond slower to temperature changes than glass capillaries. Greater wall thickness variation and capillary tapering in the plastic tubes also compromise symmetry advantages for temperature homogeneity found in glass capillaries.

The sample temperature response within plastic and glass capillaries, run under identical rapid cycling protocols, is shown in Figure 1. In contrast to glass capillaries, target temperatures are not reached in plastic capillaries. In addition, the cycle time is increased, an effect that depends on the substitute carousel, which apparently has a greater thermal mass. Whenever the denaturation or annealing temperatures are not reached, PCR failure would be expected. That is, product denaturation and primer annealing must occur for PCR amplification.

Figure 2 shows actual PCR results amplified in glass and plastic capillaries using human genomic (ACVRLI and $A P O E$ ) and plasmid (pBR322) DNA as template. Under rapid cycling conditions with momentary ("0" s) denaturation and annealing times, real-time PCR with glass capillaries shows strong amplification of all targets first observed between cycles 20-25, whereas plastic capillaries show either no fluorescence (ACVRL1 and $A P O E$ ) or a late rise in fluorescence at cycle 35 (pBR322). High-resolution melting and end-point gel analysis demonstrates specific products from the glass capillaries, and absent or low melting, small-size products from the plastic capillaries. When 5 or $10 \mathrm{~s}$ holding

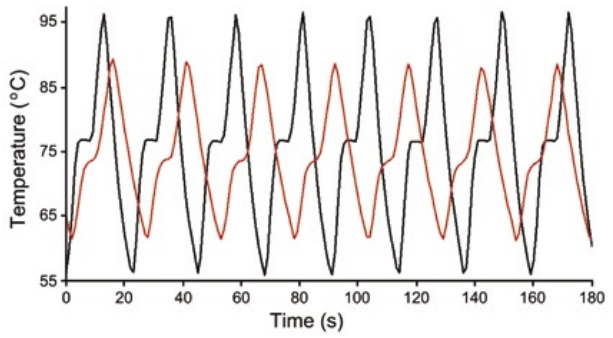

Figure 1. Sample temperature traces within glass and plastic capillaries during rapid-cycle PCR with momentary denaturation and annealing. The programmed cycling protocol was $95^{\circ} \mathrm{C}$ for $0 \mathrm{~s}, 55^{\circ} \mathrm{C}$ for $0 \mathrm{~s}$, and $75^{\circ} \mathrm{C}$ for $5 \mathrm{~s}$. The sample reaches the target temperature for each stage of PCR when glass capillaries are used (black line). However, the temperature response of samples within plastic capillaries (red line) is slower. The maximum temperature achieved in plastic capillaries is $88.7^{\circ} \mathrm{C}, 6.3^{\circ} \mathrm{C}$ away from the target of $95.0^{\circ} \mathrm{C}$. The minimum temperature achieved in plastic capillaries is $61.5^{\circ} \mathrm{C}, 6.5^{\circ} \mathrm{C}$ away from the target of $55.0^{\circ} \mathrm{C}$. The temperature range (maximum-minimum) achieved by samples in plastic capillaries is only $67 \%$ of that for samples in glass capillaries.

times were added to both denaturation and annealing segments, PCR was successful in plastic capillaries for the $A P O E$ target, but not the $A C V R L 1$ or pBR322 target. A slight increase in the apparent $\mathrm{T}_{\mathrm{m}}$ of the APOE product in plastic capillaries may result from the lower thermal conductivity of plastic and the rather rapid $0.3^{\circ} \mathrm{C} / \mathrm{s}$ melting rate used.

Figure 3 shows how long it takes samples in plastic capillaries to reach target denaturation and annealing temperatures. The sample temperature comes to within $1^{\circ} \mathrm{C}$ of the denaturation and annealing temperatures with holding times of $15-20 \mathrm{~s}$.

Table 1. Dimensions and Thermal Conductivity of Glass and Plastic Capillary Tubes

\begin{tabular}{|c|c|c|c|}
\hline \multirow[t]{2}{*}{ Property } & \multirow[t]{2}{*}{ Glass } & \multicolumn{2}{|c|}{ Plastic (Topas) } \\
\hline & & Base & Tip \\
\hline \multicolumn{4}{|l|}{ Diameter (mm) } \\
\hline Outer & 1.53 & 2.42 & 2.12 \\
\hline Inner & 1.17 & 1.81 & 1.53 \\
\hline \multicolumn{4}{|l|}{ Wall Thickness $(\mu \mathrm{m})$} \\
\hline Minimum & 174 & 235 & 220 \\
\hline Maximum & 187 & 327 & 405 \\
\hline Surface area/volume $\left(\mathrm{mm}^{-1}\right)$ & 2.80 & \multicolumn{2}{|c|}{2.17} \\
\hline Thermal conductivity $\left(\mathrm{Wm}^{-1} \mathrm{~K}^{-1}\right)$ & $0.5-1.4$ & \multicolumn{2}{|c|}{$0.12-0.15$} \\
\hline
\end{tabular}

Thermal conductivity data taken from product data sheets (Schott North America, Duryea, PA, USA, and Topas Advanced Polymers, Florence, KY, USA) 

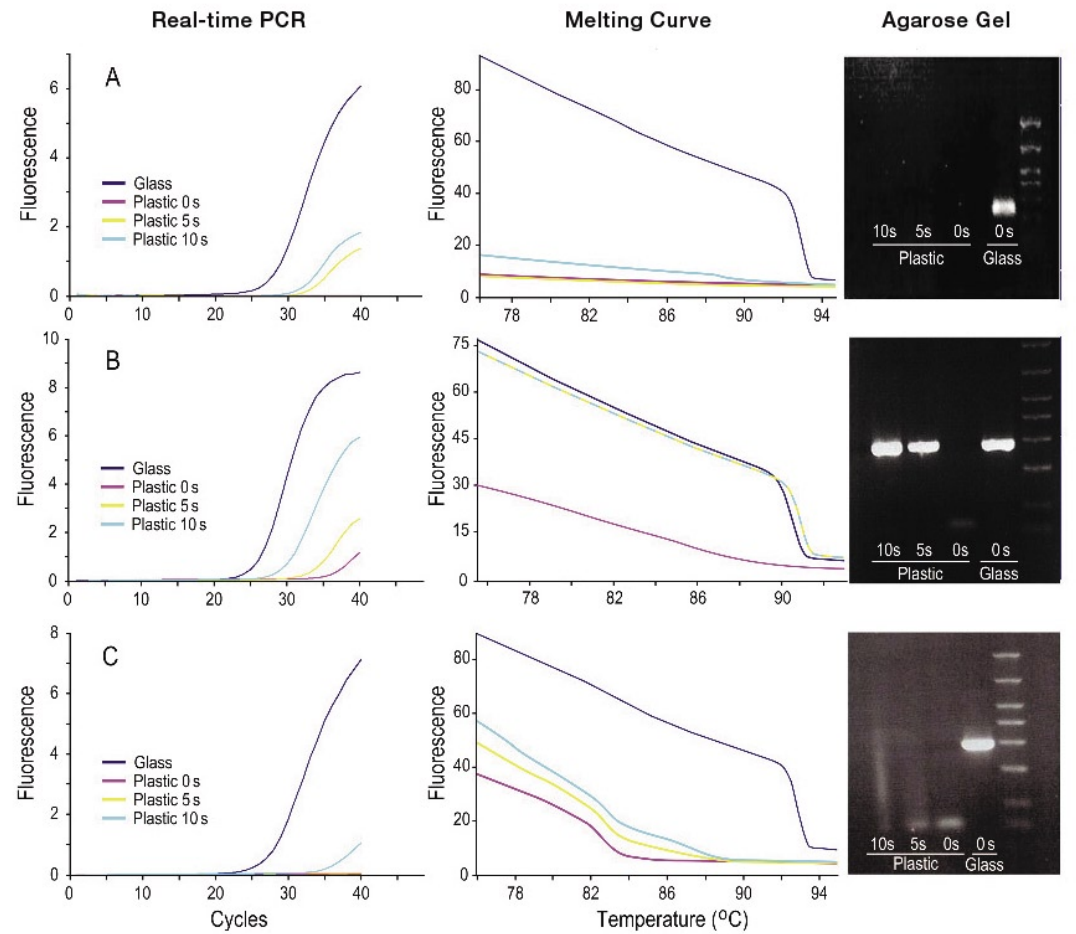

Figure 2. Real-time PCR, product melting curves, and agarose gel electrophoresis of three PCR products. Momentary ("0" s) holding times at denaturation and annealing were programmed and the results in glass (black lines) and plastic capillaries (purple) compared. Samples in plastic capillaries were also amplified with $5 \mathrm{~s}$ (yellow) and $10 \mathrm{~s}$ (blue) holding times at denaturation and annealing temperatures. A $225 \mathrm{bp} \mathrm{ACVRL1}$ gene fragment of $68 \% \mathrm{GC}$ content and $\mathrm{T}_{\mathrm{m}}$ of $93.1^{\circ} \mathrm{C}$ is shown in (A), amplified after an initial denaturation of $96^{\circ} \mathrm{C}$ for $10 \mathrm{~s}$ by 40 cycles of $68^{\circ} \mathrm{C}$ for $0 \mathrm{~s}, 75^{\circ} \mathrm{C}$ for $5 \mathrm{~s}$, and $94^{\circ} \mathrm{C}$ for 0 s. (B) shows a $279 \mathrm{bp}$ fragment of $A P O E$ with $75 \% \mathrm{GC}$ content and a $\mathrm{T}_{\mathrm{m}}$ of $90.3^{\circ} \mathrm{C}$ amplified under the same conditions as ACVRL1 except that $7 \%$ DMSO was present. (C) shows a $300 \mathrm{bp}$ fragment of pBR322 with $61 \% \mathrm{GC}$ content and a $\mathrm{T}_{\mathrm{m}}$ of $92.9^{\circ} \mathrm{C}$, amplified under the same conditions as ACVRL1 except with an annealing temperature of $57^{\circ} \mathrm{C}$. The molecular size marker (BioMarker Low, BioVentures, Murfreesboro, TN, USA) is on the right lane of each gel.

Rapid-cycle PCR instruments with the capability of " 0 " s denaturation and annealing times are precisely matched to the thermal response of the capillary. Although plastic capillaries can be used in these instruments, samples will only reach target temperatures with denaturation and annealing times of $20 \mathrm{~s}$. Because of different dimensions and thermal conductivity, the temperature/time course of the sample depends strongly on the capillary used. It is not reasonable to expect the same PCR results in glass and plastic capillaries that use the same temperature programming, particularly with rapid cycling protocols. Three PCR products of moderate length (225-300 bp) and moderately high GC content $(61 \%-75 \%)$ were studied here. Better results with plastic capillaries may be easier to obtain with lower GC content targets. However, the slower approach to annealing would be expected to always affect specificity $(3,5)$.
If concern over glass breakage requires a transfer to plastic, the need to re-optimize temperature/time parameters should be expected. Rapid-cycle PCR with momentary denaturation and annealing will fail if capillaries of slower thermal response are used. With $20 \mathrm{~s}$ denaturation and annealing times, target temperatures are reached in plastic capillaries and most products can be amplified. However, the advantages of rapid cycling, both in turnaround time (10-30 $\mathrm{min})$ and enhanced specificity (2) are largely lost. Since re-optimization is necessary when the sample vessel is changed, transfer to more conventional thermal cyclers may be preferred over operating a rapidcycle instrument with plastic capillaries that results in a dynamic temperature mismatch.

Rapid-cycle PCR with momentary denaturation and annealing requires a precise match between the temperature sensor and the sample. One possible

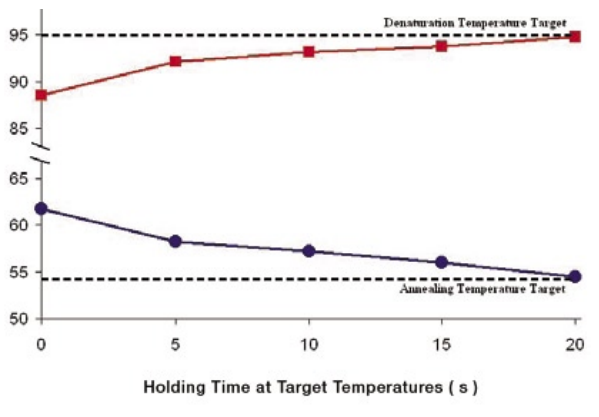

Figure 3. Denaturation and annealing holding times required for the sample to approach target temperatures in plastic capillaries. The instrument was programmed for denaturation at $95^{\circ} \mathrm{C}$ annealing at $55^{\circ} \mathrm{C}$, and extension at $72^{\circ} \mathrm{C}$ for $5 \mathrm{~s}$. The programmed holding times at denaturation and annealing target temperatures varied from 0 to $20 \mathrm{~s}$. The high and low sample temperatures reached during cycling are plotted against the holding times at denaturation and annealing.

approach is to change the temperature response of the sensor to match a new capillary. This has been done in the LightCycler 2.0 series instruments, where different sensors are used for different glass capillaries designed to hold $20 \mu \mathrm{L}$ or $100 \mu \mathrm{L}$. However, cycling speeds are still compromised with the larger capillaries because of larger sample volume, lower surface area-to-volume ratio, and increased wall thickness. Furthermore, thermal matching to a new capillary requires hardware (sensor) and/or software modifications not available for second-source capillaries. Although it may be possible to eventually develop a plastic substitute that allows rapid cycling with comparable physical rigidity, wall thickness, thermal conductivity, and uniformity of glass capillaries, it is a tall order to fill and the reason rapid cycling techniques were developed with glass capillaries $(1,3,6)$.

Many clinical, research, and industrial applications are focused on throughput rather than turnaround time and do not require rapid cycling. Sample procurement, transport, and sample preparation are often limiting. Furthermore, some hot start methods (heat activated polymerases) and decontamination procedures (uracyl deglycosylase) increase the baseline time required for PCR. Nevertheless, slower PCR is inherently less specific $(2,3,5)$ and long amplification times limit the availability of expensive real-time instruments.

Rapid-cycle PCR is an advantage whenever fast turnaround time and 
ArrayStar

shines at

straightforward
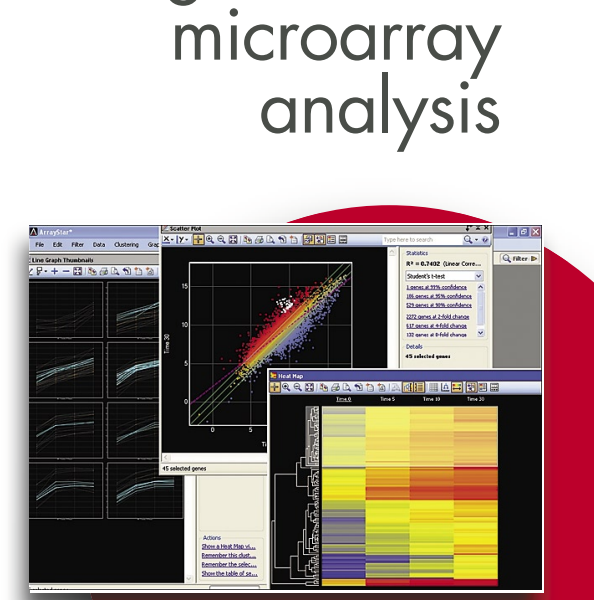

A selection of genes highlighted within simultaneous multiple

views of ArrayStar

You benefit from these features and more!

- Compatible with Affymetrix and NimbleGen software

- Works seamlessly with .txt files

- Conducts a wide range of analyses and visualizations to evaluate expression patterns

- Clusters images to identify groups of co-regulated genes

- Plots gene expression levels to view relationships with Line Graphs and Thumbnails

To receive a FREE

fully-functional trial version visit our website or contact us today.

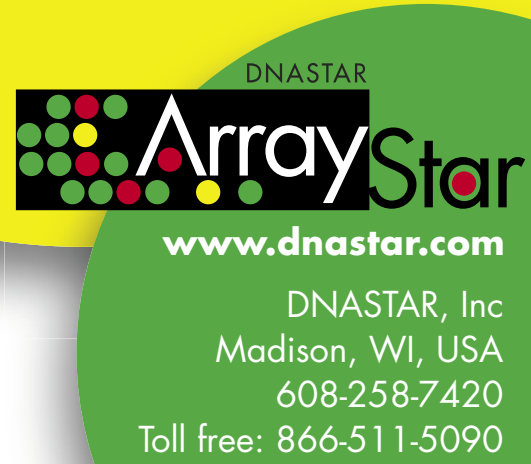

high specificity are desirable. Clinical scenarios include bacterial speciation (10), antibiotic resistance determination (11), and pharmacogenetic testing for therapeutic dosing (12). Research applications include DNA engineering requiring many sequential PCRs (13). Rapid-cycle PCR is best performed on small volume samples and is a popular technique in microdevices $(14,15)$. With larger volumes commonly used in standard equipment, rapid PCR in 10-30 min is still possible if the sample temperature is accurately controlled. Heating samples in glass capillaries with circulating air provides a unique solution for rapid-cycle PCR. However, use of plastic capillaries in systems matched to glass capillaries requires empirical optimization at slower speeds with compromises in turnaround time, temperature homogeneity, and PCR specificity.

\section{ACKNOWLEDGMENTS}

This work was supported by National Institutes of Health (NIH) grants (nos. GM72419 and GM73396).

\section{COMPETING INTERESTS \\ STATEMENT}

Aspects of rapid-cycle PCR, real-time $P C R$, and melting analysis are licensed from the University of Utah (Salt Lake City) to Idaho Technology and from Idaho Technology to Roche Applied Science. C.T.W. holds equity interest in Idaho Technology. D.D. is an employee of Idaho Technology.

\section{REFERENCES}

1. Wittwer, C.T., G.C. Fillmore, and D.J. Garling. 1990. Minimizing the time required for DNA amplification by efficient heat transfer to small samples. Anal. Biochem. 186:328331.

2. Wittwer, C.T. and D.J. Garling. 1991. Rapid cycle DNA amplification: time and temperature optimization. BioTechniques 10:76-83.

3. Wittwer, C.T., G.B. Reed, and K.M. Ririe. 1994. Rapid cycle DNA amplification, p. 174181. In Mullis, I.K., F. Ferre, and R. Gibbs (Eds.), The Polymerase Chain Reaction. Birkhauser, Boston, MA.

4. Wittwer, C.T. and M.G. Herrmann. 1999. Rapid thermal cycling and PCR kinetics, p. 211-229. In Innis, M., D. Gelfand, and J. Sninsky (Eds.), PCR Methods Manual. Academic Press, San Diego, CA.
5. Wittwer, C.T., B.C. Marshall, G.H. Reed, and J.L. Cherry. 1993. Rapid cycle allelespecific amplification: studies with the cystic fibrosis delta F508 locus. Clin. Chem. 39:804809.

6. Wittwer, C.T., K.M. Ririe, R.V. Andrew, D.A. David, R.A. Gundry, and U.J. Balis. 1997. The LightCycler: a microvolume multisample fluorimeter with rapid temperature control. BioTechniques 22:176-181.

7. Vandersteen, J.G., P. Bayrak-Toydemir, R.A. Palais, and C.T. Wittwer. 2007. Identifying common genetic variants by high-resolution melting. Clin. Chem. 53:1191-1198.

8. Poulson, M.D. and C.T. Wittwer. 2007 Closed-tube genotyping of apolipoprotein E by isolated-probe PCR with multiple unlabeled probes and high-resolution DNA melting analysis. BioTechniques 43:87-91

9. Highsmith, W.E., Jr., Q. Jin, A.J. Nataraj, J.M. O'Connor, V.D. Burland, W.R. Baubonis, F.P. Curtis, N. Kusukawa, and M.M. Garner. 1999. Use of a DNA toolbox for the characterization of mutation scanning methods. I: construction of the toolbox and evaluation of heteroduplex analysis. Electrophoresis 20:1186-1194.

10. Cheng, J.C., C.L. Huang, C.C. Lin, C.C. Chen, Y.C. Chang, S.S. Chang, and C.P. Tseng. 2006. Rapid detection and identification of clinically important bacteria by highresolution melting analysis after broad-range ribosomal RNA real-time PCR. Clin. Chem. 52:1997-2004.

11. Slinger, R., D. Bellfoy, M. Desjardins, and F. Chan. 2007. High-resolution melting assay for the detection of gyrA mutations causing quinolone resistance in Salmonella enterica serovars Typhi and Paratyphi. Diagn. Microbiol. Infect. Dis. 57:455-458.

12. Carlquist, J.F., J.T. McKinney, Z.P. Nicholas, J.L. Clark, S.F. Kahn, B.D. Horne, J.B. Muhlestein, H.T. May, et al. 2007. Rapid melting curve analysis for genetic variants that underlie inter-individual variability in stable warfarin dosing. J. Thromb. Thrombolysis. [Epub ahead of print, July 29, 2007].

13. Brown, R.A., M.J. Lay, and C.T. Wittwer. 1998. Rapid cycle amplification for construction of competitive templates, p. 57-70. In Horton, R.M. and R.C. Tait (Eds.), Genetic Engineering with PCR. Horizon Scientific Press, Norfolk, VA.

14. Roper, M.G., C.J. Easley, and J.P. Landers. 2005. Advances in polymerase chain reaction on microfluidic chips. Anal. Chem. 77:38873893.

15. Zhang, C. and D. Xing. 2007. Miniaturized PCR chips for nucleic acid amplification and analysis: latest advances and future trends. Nucleic Acids Res. 35:4223-4237.

Received 13 October 2007; accepted 5 December 2007.

Address correspondence to Carl T. Wittwer, Department of Pathology. 50 North Medical Drive, University of Utah, Salt Lake City, UT, 84132, USA. e-mail: carl. wittwer@path.utah.edu

To purchase reprints of this article, contact: Reprints@BioTechniques.com 